\title{
Poliovirus Resistant Cells Derived from HeLa Cells
}

\author{
MASANOBU CHINAMI, EIJI NAKAMURA*, SHINICHI KAKISAKO, \\ BING XU AND MASAHISA SHINGU
}

\author{
Department of Virology and ${ }^{*}$ Department of Internal Medicine, Kurume University \\ School of Medicine, Kurume, 830 Japan
}

Received for publication August 23, 1986

\begin{abstract}
Summary: A cell line from HeLa cells was established, which did not show a cytopathogenic effect (CPE) to poliovirus infection. The cell line, designated PRHC-1, did not regain susceptibility after more than 100 passages for one year. The poliovirus type 1-Mahoney, Sabin-2 and Sabin-3 also were not infective to PRHC-1 cells, however a bovine enterovirus (MZ-468) was. No binding activity of PRHC-1 cells to poliovirus Sabin-1 was observed. A chromosome analysis demonstrated no significant difference between PRHC-1 and its parental HeLa cells. Immunofluorescence staining demonstrated no persistent infection in PRHC-1 cells.
\end{abstract}

Key words: receptor deficiency - chromosome analysis - no persistency bovine enterovirus - immunofluorescence staining

\section{Introduction}

Picornavirus infection is initiated after binding to cellular receptors (Dales, 1973). Only primate and human cells are known to be susceptible to poliovirus (Holland, 1964). Soloviev et al. (1968) described cell lines that were resistant to poliovirus, many years ago. Miller et al. (1974) suggested that the gene for the poliovirus receptor is located on the 19 th human chromosome. No further genetic analysis of the receptor has been done. The biochemical properties of the receptors also have not been clearly elucidated. Thus the establishment of a cell line resistant to poliovirus would be a significant step towards the characterization of the receptor and the elucidation of the infective mechanism of the poliovirus.

\section{Materials and Methods}

HeLa-S3 cells were cultured in a Minimum essential medium (GIBCO) containing $10 \%$ calf serum. Resistant HeLa cells were produced by inoculation of cell monolayers with poliovirus Sabin-1 at moi of 1. 0 . Most of the cultured cells were destroyed within two days after infection. A few surviving cells were further cultivated in a $\mathrm{CO}_{2}$ incubator. The established cell line, designated as PRHC-1, was passaged every two or three days for one year (over one hundred passages). Poliovirus Sabin-1, poliovirus Sabin-2, poliovirus Sabin-3, poliovirus type- 1 Mahoney strain and bovine enterovirus (MZ-468) were propagated in HeLa cell monolayers at moi of 1. 0 . The 110 th passage cells were used for the following experiments unless otherwise stated. HeLa and PRHC-1 cells were infected at moi of 1.0 to the above viruses for examination of susceptibility. The cell culture supernatant, freezethawed materials and viable whole PRHC cells were 
added to parental HeLa cells and observed for five days to determine whether any persistent infection of the poliovirus in PRHC-1 cells was present. Completely monolayered HeLa and PRHC-1 cells $(3 \times$ $10^{6}$ ) in $5 \mathrm{~cm}$ diameter round dishes (Nunc, Denmark) were inoculated with $7.8 \times 10^{3}$ pfu of poliovirus Sabin-1 in PBS ( $\mathrm{pH} 7.4$ ) and incubated at $24^{\circ} \mathrm{C}$ for two hours with stirring every $15 \mathrm{~min}$. Non-adsorbed virus was titrated by plaque assay. ddY mice were immunized with the poliovirus, which was propagated in African Green Monkey Kidney (GMK) cells and purified with $\mathrm{CsCl}$, as previously reported (Chinami and Shingu, 1985). The IgG fraction was obtained in the same way. Monolayered HeLa and PRHC-1 cells were infected with the poliovirus Sabin-1 at moi of 10 and incubated at $37^{\circ} \mathrm{C}$ for $14 \mathrm{hrs}$. The cells were washed twice with saline and fixed with acetone. After fixation, the cells were washed with saline and incubated with IgG antibody against the poliovirus at $4^{\circ} \mathrm{C}$ for $30 \mathrm{~min}$. After a second washing with saline, the cells were incubated with the second antibody, FITC-labeled goat antimouse IgG, purchased from Cooper Biomedical, USA. HeLa cells and PRHC-1 cells (passage number $83 \mathrm{th}$ ) were cultured in MEM containing $10 \%$ calf serum for 6 hrs, after which the mediums were changed to PBS containing $2 \mu \mathrm{g}$ of colchicin. The cells were incubated for $1 \mathrm{hr}$ and fixed with Carnoy solution and stained with Giemsa solution for chromosome analysis.

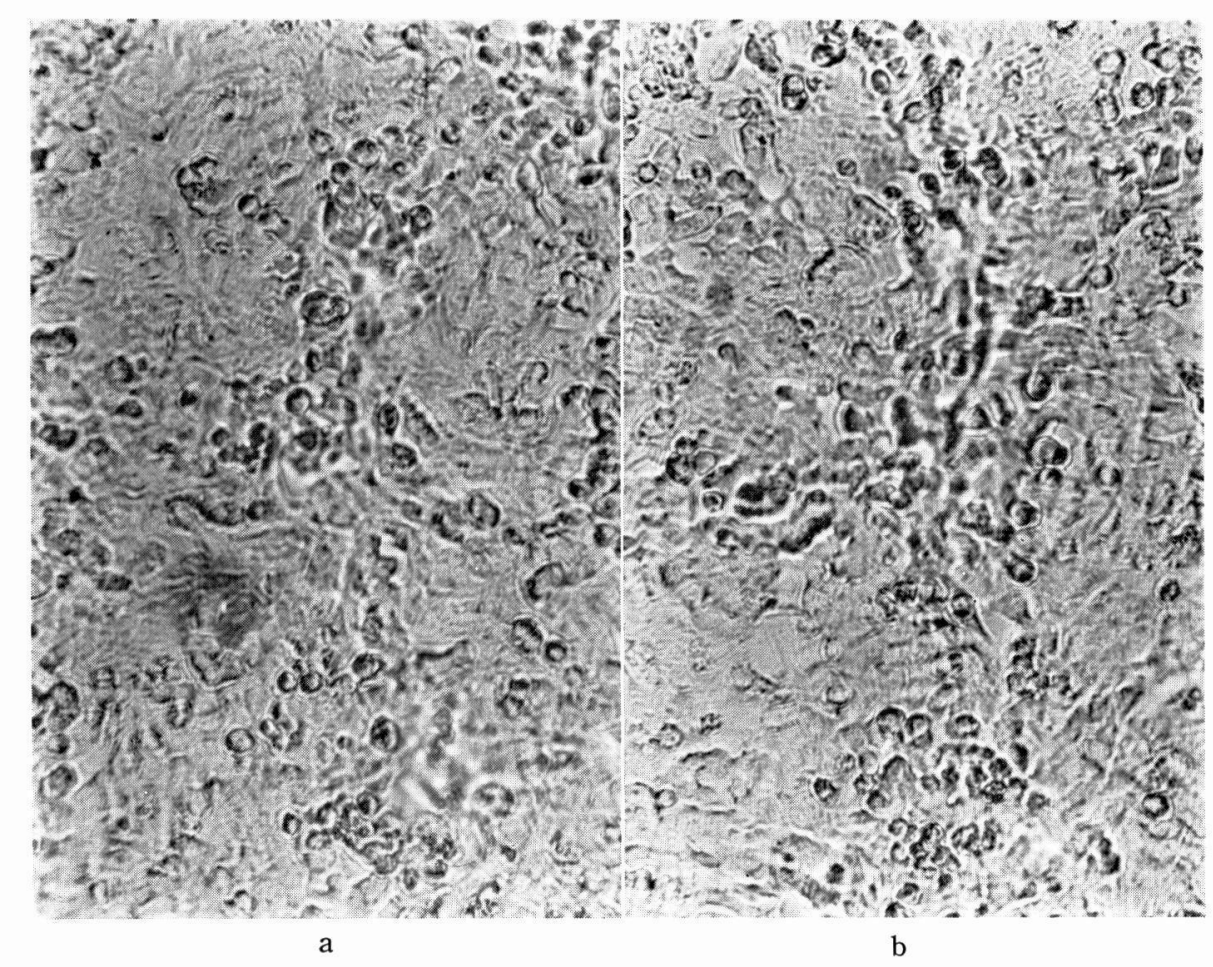

Fig. 1. Photomicrographs $(\times 100)$ of HeLa (a) cells and PRHC-1 cells (b). 


\section{TABLE 1}

Susceptibility of PRHC-1 cells to viruses. Monolayered PRHC-1 cells were infected with viruses at moi of 1.0 and observed with a microscope for 5 days.

\begin{tabular}{cc}
\hline Virus & $\begin{array}{c}\text { Cytopathogenic effect } \\
\text { in PRHC cells }\end{array}$ \\
\hline $\begin{array}{l}\text { Poliovirus Sabin-1 } \\
\text { Poliovirus type-1 } \\
\text { Mahoney }\end{array}$ \\
Poliovirus Sabin-2 \\
Poliovirus Sabin-3 \\
$\begin{array}{l}\text { Bovine enterovirus } \\
\text { (MZ-468) }\end{array}$ \\
\hline
\end{tabular}

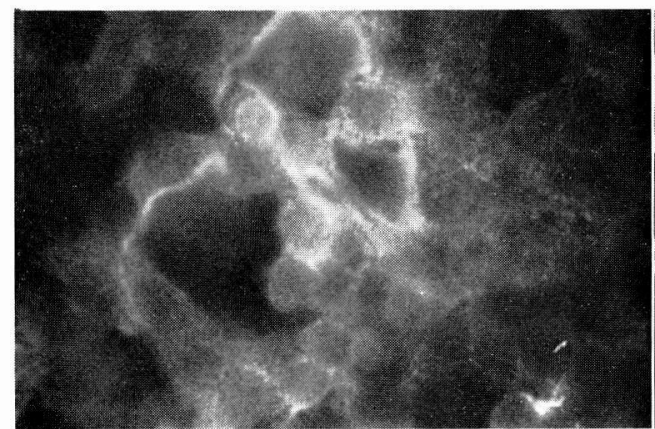

A

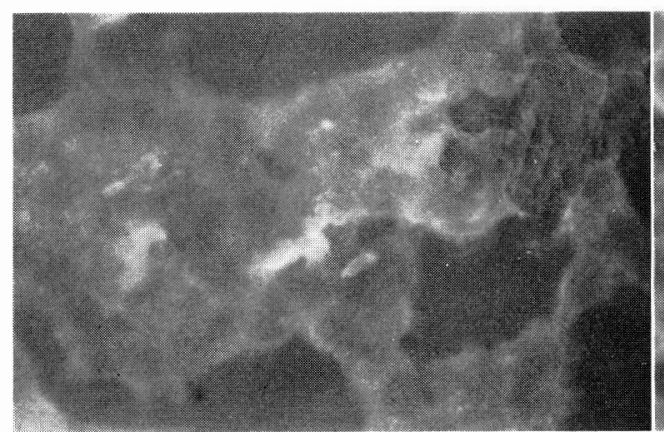

C

\section{TABLE 2}

Binding activities of poliovirus Sabin-1 to HeLa and PRHC-1 cells. Completely monolayered cells were inoculated with $7.8 \times$ $10^{3}$ pfu in PBS (pH 7.4) at $24^{\circ} \mathrm{C}$ for two hours. Non-adsorbed virus was titrated by plaque assay. The data from 8 samples was statistical analyzed.

\begin{tabular}{cc}
\hline Cells & Binding Ratio \\
\hline HeLa cells & $50( \pm 6) \%$ \\
PRHC -1 cells & $5( \pm 7) \%$
\end{tabular}

( ): standard deviation

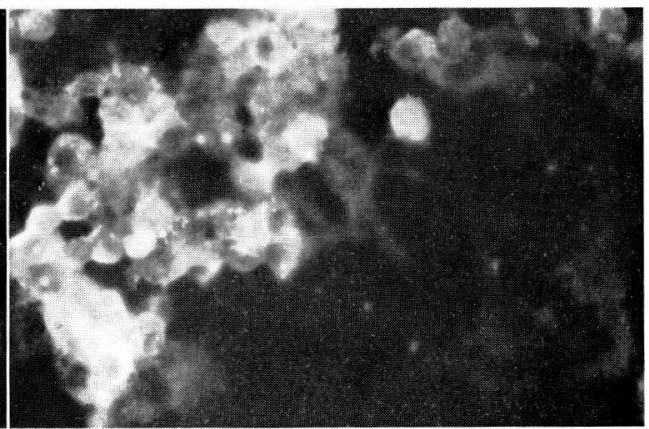

B

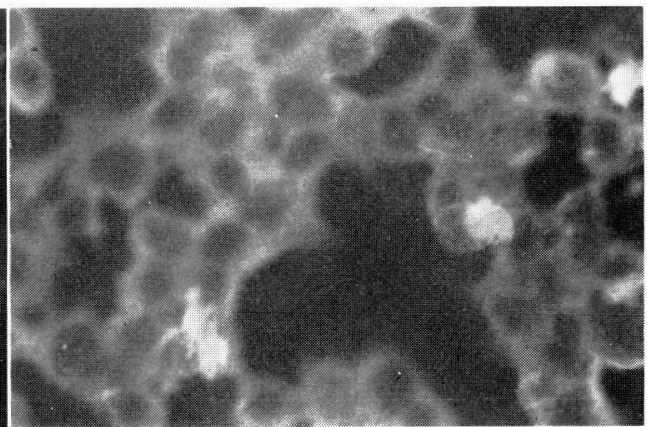

$\mathrm{D}$

Fig. 2. Photographs of indirect immunofluorescence staining of HeLa and PRHC-1 cells infected or not infected with poliovirus Sabin-1. HeLa and PRHC-1 cells, monolayered on cover glasses, were infected at moi of 10 and incubated at $37^{\circ} \mathrm{C}$ for $14 \mathrm{hrs}$. After fixation with acetone, IgG antibody for poliovirus Sabin-1 and fluorescinated goat anti-mouse IgG were reacted as described in the methods. Non virus-infected samples of both cells were used as controls. A, control HeLa cells. B, virus infected HeLa cells. C, control PRHC-1 cells. D, virus-infected PRHC-1 cells. 


\section{Results}

Microscopic observations of the PRHC-1 cells have not demonstrated any prominent change at any passage since the cell line was established. A microscopic picture of PRHC-1 cells after 110 passages is shown in Fig. 1 and compared with parental HeLa cells. The susceptibility of the PRHC-1 cells to poliovirus Sabin-1 and other related viruses are shown in Table 1 . Only a bovine enterovirus (MZ-468) displayed susceptibility for the cells. The binding activities of the poliovirus Sabin-1 to the PRHC-cells and HeLa cells were examined and a decreased binding activity in PRHC-1 cells was observed (Table 2). The level of indirect immunofluorescence staining of PRHC-1 cells, which were infected with poliovirus Sabin-1, was almost the same as that for control HeLa cells (Fig. 2).

\section{Discussion}

In the processes of picornavirus infection, the following steps are known to be involved. Adsorption of the virus particle to a cellular receptor, degradation of the virion, replication of the RNA, synthesis of viral protein and release of the virion into the extra-cellular space after assembly of viral proteins. If CPE does not appear in vitro after viral infection, one or more of the steps described above must be affected. The PRHC-1 cells were not changed, morphologically. There are many reports about persistent infections which have no cytopathogenic effect (CPE) (Gibson and Righthand, 1985; Vallbracht et al. 1984). Thus it was necessary to confirm whether there was a persistent infection by the poliovirus Sabin-1 in the PRHC-1 cells. Inoculation of supernatant, cell extracts or whole viable PRHC-1 cells into HeLa cell monolayers did not show $\mathrm{CPE}$ or any infectious centers, demon- strating that no persistency exists in the PRHC-1 cells (data not shown). Further the fact that no prominent viral antigens were demonstrated in PRHC-1 cells by immunofluorescence staining suppors the above results. It is concluded that the escape from CPE by the PRHC-1 cells is not due to the persistence of the poliovirus. To examine the ability of the PRHC-1 cells to bind poliovirus Sabin-1, a binding assay of the virus to the cells was performed by plaque titration. In this experiment, the number of cells far exceeded the number of the input virus, and the number of receptor sites was therefore greater than the number of input virus particles even if one assumes that $1 \mathrm{pfu}$ is over $10^{3}$ particles (Rueckert, 1976). As shown in Table 2, a small amount of binding activity of PRHC-1 cells to the virus was observed, probably due to nonspecific binding. This result suggests that the resistancy of PRHC-1 cells to poliovirus Sabin-1 is caused by an inability to bind the virus, possibly by the mechanism described by Kimura et al. (1985) for the influenzavirus. That is, some structural change in the receptor may have occurred. Loss of susceptibility of PRHC-1 cells to poliovirus Sabin-2, poliovirus Sabin-3 and poliovirus type 1-Mahoney, which are all closely related to poliovirus Sabin-1, indicates that all these receptors have some degree of structural similarity. This concept was supported by the results of a binding interference assay using poliovirus type-1, type-2 and type-3 (Crowell, 1976). Susceptibility of a bovine enterovirus (MZ468) to PRHC-1 cells remained, which means that its receptor is quite different from the receptors for the polioviruses. At each metaphase, chromosomes from 20 cells were examined. The number of chromosomes of HeLa and PRHC-1 cells ranged from 50-68 and 47-66, respectively (hyperdiploid). Similar abnormal structures in chromosomes No. 1 and No. 3 were found. Many marker chromosomes were 
present in both cell lines and the 19 th chromosome was also found in both of cell lines. In conclusion, the lack of remarkable differences in the chromosomes indicates that PRHC-1 cells were derived from parental HeLa cells. But confirmation of the existence of the 19 th chromosome in PRHC-1 cells is not compatible with the concept of Miller et al. (1974). We are now clarifying the mechanism for the resistance of PRHC-1 cells to poliovirus by comparing the biochemical properties of membrane fractions of both cells.

Acknowledgment: The author would like to thank Mr. Gary R. Wyckoff for correcting the manuscripts.

\section{References}

Chinami, M. and Shingu, M. (1985). Cytoflow metric analysis and binding pattern and a new kinetic constant for an enterovirus and receptor. Kurume Med. J. 32, 21-27.

CROWELl, R. L. (1976). Comparative genetic characteristics of picornavirus receptor interactions. In Cell membrane receptors for viruses, antigens, antibodies, polypeptide hormone and small molecules. ed. Beers, R.F. and Basset,
E.G. pp. 179-202, New York: Raven Press.

DALES, S. (1973). Early events in cell-animal virus interactions. Bacteriol. Rev. 37, 103135.

Gibson, J. P. and Righthand, V.F. (1985). Persistence of Echovirus 6 in cloned human cells. J. Virol. 54, 219-223.

Holland, J. J. (1964). Enterovirus entrance into specific host cells, and alterations of cell protein and nucleic acid synthesis. Bacteriol. Rev. 28, 3-10.

Kimura, Y., YoKochi, T., Miyadai, T., Yoshida, K., Yoкоo, J. and Matsumoto, K. (1985). Characterization of a porcine kidney cell line resistant to influenza virus infection. J. Virol. 53, 980-983.

Miller, D. A., Miller, O. J., Dev, V.G., Hashim, S., Tantravahi, R. Medrano, L. and Green, H. (1974). Human chromosome 19 carries a poliovirus receptor gene. Cell, 1, 167-173.

Rueckert, R.R. (1976). On the structure and morphogenesis of picornavirus. In Comprehensive virology. 6, ed. Fraenkel-Conrat, $\mathrm{H}$. pp. 131-213. New York: Plenum.

Soloviev, V.D., Krispin, T.I., Zaslavsky, V.G. and Agol, V.I. (1968). Mechanism of resistancy to enteroviruses of some primate cells in tissue culture. J. Virol. 2, 553-557.

Vallbracht, A., Hofmann, L., Wurster, K.G. and FleHMig, B. (1984). Persistent infection of human fibroblasts by hepatitis A virus. J. Gen. Virol. 65, 609-615. 